\title{
PERSONALITY AND THE CREATIVITY OF FRONTLINE SERVICE EMPLOYEES: EXPLORING QUADRATIC AND MODERATING EFFECTS
}

\author{
Carlos M. P. Sousa, Durham University, UK \\ Filipe Coelho, University of Coimbra, Portugal \\ Cristiana Lages, Loughborough University, UK
}

\begin{abstract}
Previous studies investigated the relationship between the five factor model of personality and creativity. However, we go beyond this model and consider additional personality traits that have recently been investigated in the marketing literature as influencers of employee behavior, namely competitiveness, materialism, need for learning, and need for activity (see Brown, Mowen, Donavan, and Licata, 2002; Harris, Mowen, and Brown, 2005).

Additionally, past research has focused on linear effects, but we examine both linear and non-linear effects of personality, given the mounting evidence that personality has non-linear effects on other employee behaviors. Finally, considering the evidence on the interaction effect of personal and contextual characteristics in creativity studies (e.g., Oldham and Cummings, 1996), we also investigate the moderating effect of job autonomy on the personality/creativity relationship. Autonomy constitutes an important contextual factor, reflecting the nature of tasks and the decision latitude supervisors have passed on to subordinates.
\end{abstract}

The results indicate that four personality traits have non-linear effects, and that autonomy moderates the effects of some personality traits.

References Available Upon Request 